\title{
Calcified Nodules Projecting into the Popliteal Artery Successfully Treated Through the Use of Myocardial Biopsy Forceps
}

\author{
Takenobu Shimada, ${ }^{1}$ MD, Tsuyoshi Goto, ${ }^{1}$ MD, Shunsuke Kubo, ${ }^{1}$ MD, Seiji Habara, ${ }^{1}$ MD, \\ Hiroyuki Tanaka, ${ }^{1}$ MD and Kazushige Kadota, ${ }^{1}$ MD
}

\begin{abstract}
Summary
A 69-year-old man on maintenance dialysis underwent endovascular therapy for a refractory wound due to critical limb ischemia in the popliteal and below-knee arteries. Because of bulky calcified nodules projecting into the popliteal artery, conventional balloon angioplasty alone did not seem to provide a sufficient lumen area, and we ablated the calcified nodules by using myocardial biopsy forceps for lesion preparation. Under roadmap guidance, we repeated ablation 39 times to carefully tear off the bulky calcified nodules, and subsequently performed balloon angioplasty in the popliteal artery. Neither flow-limiting dissection nor perforation occurred, and a sufficient lumen area was obtained. After treatment of the popliteal artery, conventional endovascular therapy was performed in the below-knee arteries. One month later, the wound was fully epithelialized. We report a case of critical limb ischemia with calcified nodules projecting into the popliteal artery, in which the use of myocardial biopsy forceps led to good results.
\end{abstract}

(Int Heart J 2018; 59: 660-663)

Key words: Peripheral artery disease, Endovascular therapy, Calcification, Atherectomy device, Debulking device

$\mathrm{E}$ ndovascular therapy is widely accepted as a treatment of peripheral artery disease with femoropopliteal lesions. ${ }^{1,2)}$ The treatment of severely calcified lesions, however, remains challenging in terms of lesion modification and long-term patency, and atherectomy devicesreported to be effective ${ }^{3)}$ are currently unavailable in Japan. We report a case of critical limb ischemia with calcified nodules projecting into the popliteal artery that was successfully treated by using myocardial biopsy forceps.

\section{Case Report}

A 69-year-old man was admitted for peripheral artery disease presenting as critical limb ischemia (Rutherford class 5). His left toe was gangrenous and was infected. He was on maintenance dialysis and had a past history of coronary artery bypass grafting.

Angiography showed severe stenoses in the superficial femoral, popliteal, and below-knee arteries. After endovascular therapy for superficial femoral artery lesions, minor amputation was performed. Because the edge of the transmetatarsal amputation did not fully heal (Figure 1A), additional endovascular therapy for the popliteal and below-knee artery lesions was performed. Surgical procedure was not considered to be suitable because of the risk of general anesthesia due to severe chronic obstructive pulmonary disease.
Angiography and ultrasonography showed bulky calcified nodules projecting into the popliteal artery (Figures 2, 3A). The anterior tibial and peroneal arteries were occluded, and the posterior tibial artery alone was free from stenosis (Figure 6A).

Initially, we treated the popliteal artery lesion. Because conventional balloon angioplasty alone did not seem to provide the initial optimal dilation, ablation using myocardial biopsy forceps (Cordis, Miami Lakes, FL, USA) (Figure 4B) was performed. A 6Fr guiding-sheath was antegradely inserted from the ipsilateral side, and biopsy forceps were advanced to the popliteal artery lesion. The calcified nodules were carefully ablated under the roadmap functionality (RoadmapPro, Philips Healthcare, Best, The Netherlands) guidance to avoid vessel wall perforation (Figure 5). Additionally, contrast medium was injected at intervals to obtain clear images in order to avoid perforation. For the purpose of distal protection, Filtrap ${ }^{\circledR}$ embolic protection filter (Nipro, Osaka, Japan) was deployed at the distal portion of the target lesion during the procedure. Ablation was performed 39 times in total (Figure 4A) followed by balloon angioplasty using a 5.0-mm semicompliant balloon catheter. Neither flow-limiting dissection nor perforation occurred after the procedure, and a sufficient lumen area was obtained (Figure 3B).

After ablation using biopsy forceps, passing a 0.014inch hard wire through the anterior and posterior tibial ar-

From the ${ }^{1}$ Department of Cardiology, Kurashiki Central Hospital, Okayama, Japan

Address for correspondence: Takenobu Shimada, MD, Department of Cardiology, Kurashiki Central Hospital, 1-1-1 Miwa, Kurashiki, Okayama 710-8602, Japan. E-mail: takeboyism@gmail.com

Received for publication April 12, 2017. Revised and accepted July 5, 2017

Released in advance online on J-STAGE April 6, 2018.

doi: 10.1536/ihj.17-216

All rights reserved by the International Heart Journal Association. 
teries, we performed conventional balloon angioplasty, which markedly improved blood flow without any flowlimiting dissection (Figure 6B). No distal embolism due to ablation of calcified nodules was observed in the belowknee artery. One month later, his wound was fully epithelialized (Figure 1B).

\section{Discussion}

Conventional balloon angioplasty is occasionally not sufficiently effective for the treatment of calcified lesions, especially those in the popliteal artery. We experienced a case of bulky calcified nodules projecting into the popliteal artery and successfully ablated them by using myocardial biopsy forceps.
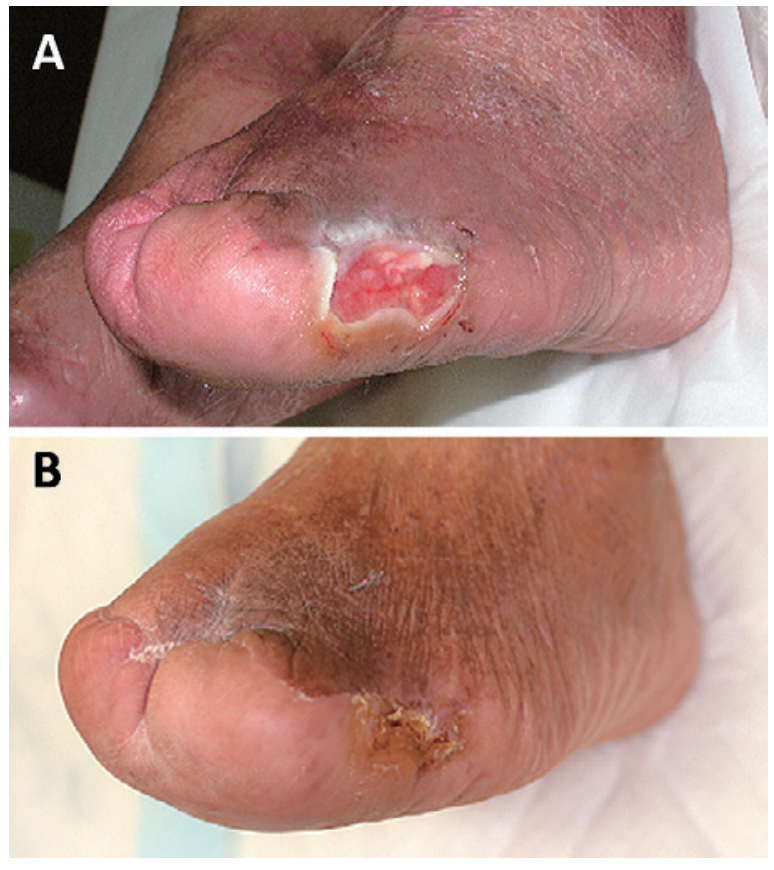

Figure 1. Wound appearance of the left toe. A: Pre-procedure. The edge of the transmetatarsal amputation did not fully heal. B: Post-procedure. The wound was epithelialized completely.
A rotational atherectomy device such as Jetstream Navitus ${ }^{\circledR}$ system (Bayer Health Care, Whippany, NJ, USA $)^{4)}$ and a directional cutting device such as SilverHawk $^{\circledR}$ catheter (Covidien, Plymouth, MN, USA) ${ }^{5)}$ are effective, but are currently unavailable in Japan. Also, atherectomy devices sometimes slip in eccentric calcified lesions or do not work sufficiently in very severely calcified lesions. ${ }^{6}$ Treatment using biopsy forceps may be an alternative for lesions that cannot be treated using the abovementioned atherectomy devices. In addition, it is important to note that our method includes an off-label use of treatment devices.

When using biopsy forceps to treat calcified lesions, the two major risks are perforation and distal embolism. Although no complication occurred in this case, prevention and early detection of complications are important. To avoid perforation, the biopsy forceps should be pulled with great caution so as not to damage the vessel wall. In this case, ablation of the calcified nodules was performed under roadmap guidance which clearly showed the motions of background images and the vessel wall being pulled by the biopsy forceps. While conventional angiographic guidance requires a large amount of contrast medium to observe vessel wall motion, roadmap guidance requires a much smaller amount, and the amount used in this procedure was only $72 \mathrm{cc}$.

Moreover, the use of biopsy forceps might have the potential to become a method for retrieving thrombi or a bail-out technique for a crucial situation such as a guidewire or a balloon being split or entrapped. To the best of our knowledge, only one case of an effective use of biopsy forceps as a bail-out technique has been reported so far, ${ }^{7)}$ and therefore further investigation is needed.

\section{Conclusion}

We described a case of critical limb ischemia with calcified nodules projecting into the popliteal artery that was successfully treated by the use of myocardial biopsy forceps.
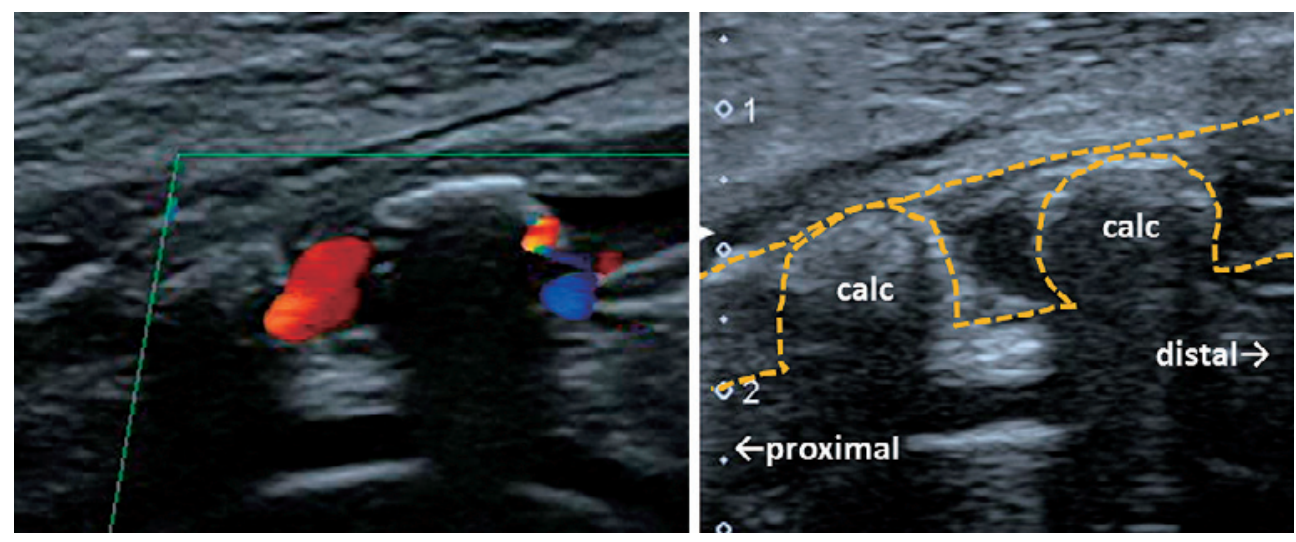

Figure 2. Ultrasonography showed severe stenosis in the popliteal artery, attributed to the calcified nodules projecting inward. 

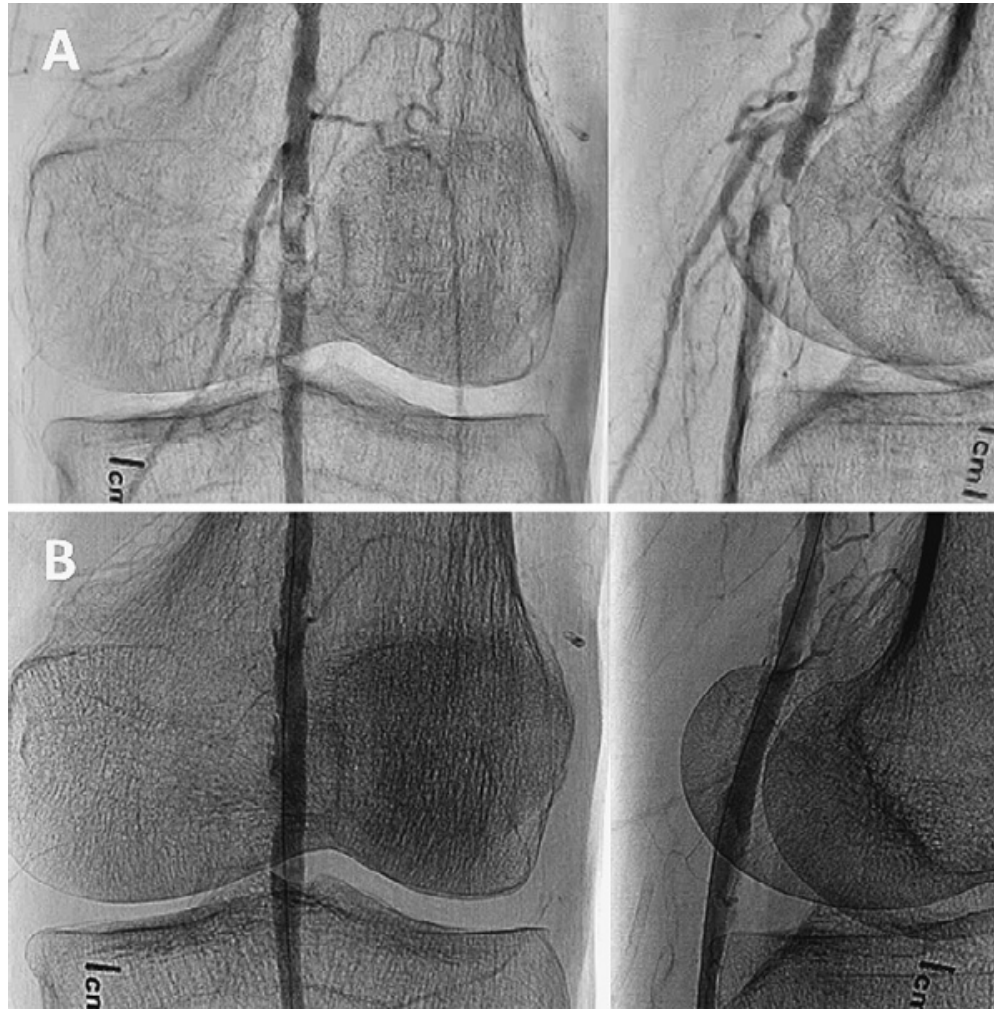

Figure 3. Angiography of the popliteal artery. A: Pre-procedure. Bulky calcified nodules projecting into the popliteal artery. B: Post-procedure. There was neither flow-limiting dissection nor perforation, and a sufficient lumen area was obtained. The left side is an antero-posterior (AP) view, and the right side is a left anterior oblique $60^{\circ}$ view.
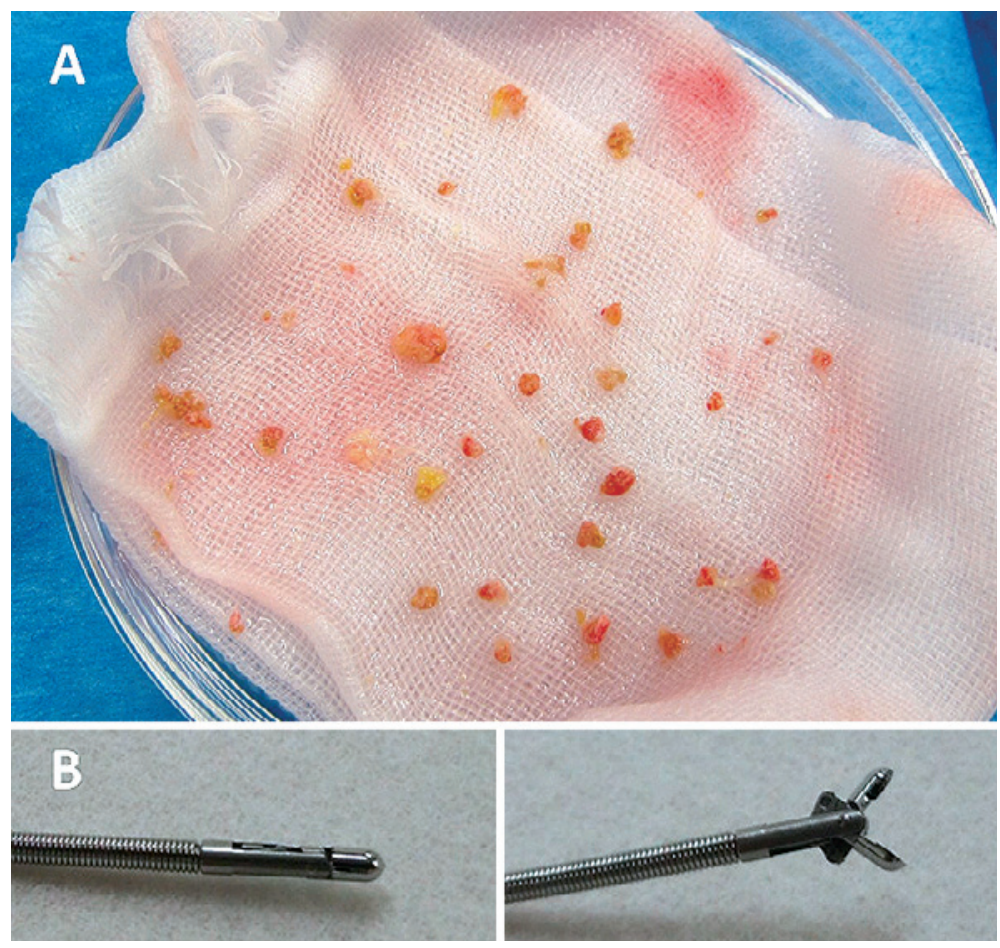

Figure 4. A: Calcified nodules extracted from the popliteal artery. B: Myocardial biopsy forceps used in this case 


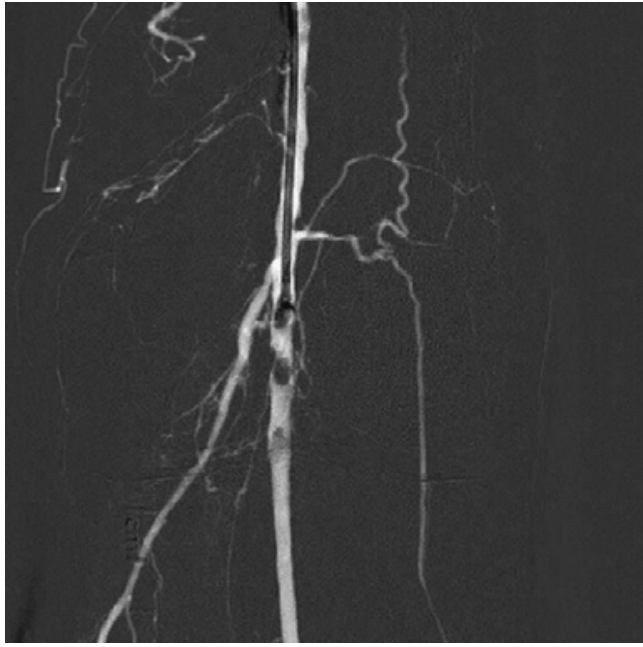

Figure 5. Angiography of ablation of the calcified nodules under roadmap functionality guidance

\section{Acknowledgment}

We are grateful to Miho Kobayashi for her secretarial assistance.

\section{Disclosures}

Conflicts of interest: The authors have no financial conflicts of interest to declare.

\section{References}

1. Norgren L, Hiatt WR, Dormandy JA, Nehler MR, Harris KA, Fowkes FG. Inter-Society Consensus for the Management of Peripheral Arterial Disease (TASC II). Eur J Vasc Endovasc Surg 2007; 33 (Suppl 1): S1-75.

2. Tendera M, Aboyans V, Bartelink ML, et al. ESC Guidelines on the diagnosis and treatment of peripheral artery diseases: Document covering atherosclerotic disease of extracranial carotid and vertebral, mesenteric, renal, upper and lower extremity arteries: the Task Force on the Diagnosis and Treatment of Periphera Artery Diseases of the European Society of Cardiology (ESC). Eur Heart J 2011; 32: 2851-906.

3. Quevedo HC, Arain SA, Ali G, Abi NR. A critical view of the peripheral atherectomy data in the treatment of infrainguinal arterial disease. J Invasive Cardiol 2014; 26: 22-9.

4. Zeller T, Krankenberg H, Steinkamp H, et al. One-year outcome of percutaneous rotational atherectomy with aspiration in in-
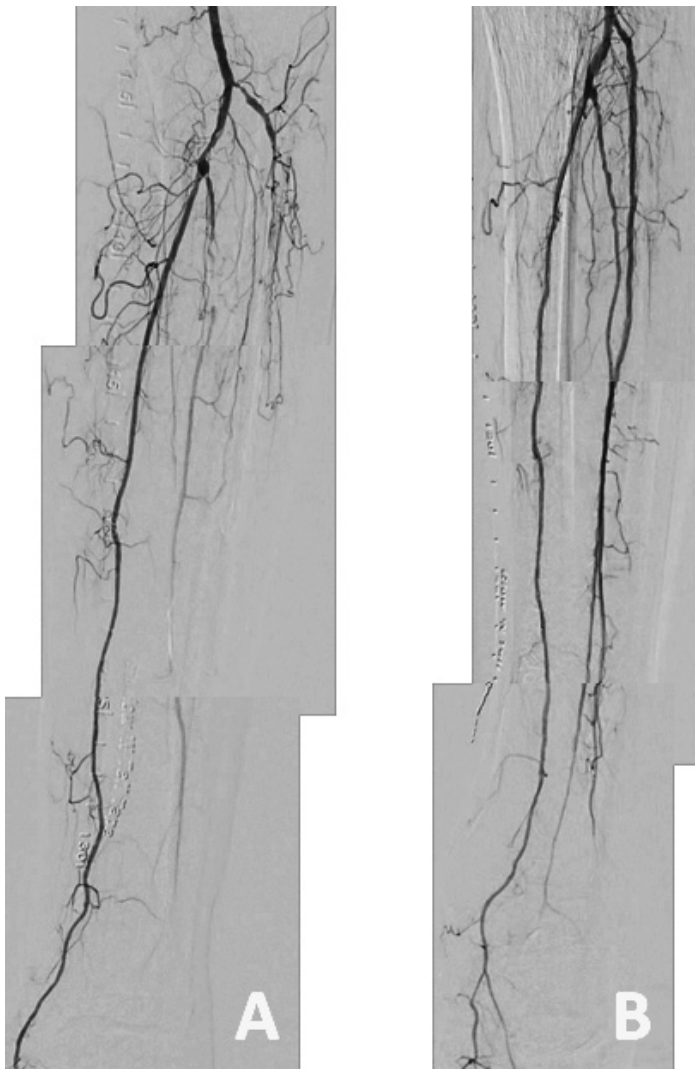

Figure 6. Angiography of the below-knee artery. A: Pre-procedure. The anterior tibial artery and peroneal artery were occluded. B: Postprocedure. Blood flow markedly improved without any flow-limiting dissection. No distal embolism due to ablation of calcified nodules was observed in the below-knee artery.

frainguinal peripheral arterial occlusive disease: the multicenter pathway PVD trial. J Endovasc Ther 2009; 16: 653-62.

5. McKinsey JF, Zeller T, Rocha-Singh KJ, Jaff MR, Garcia LA. Lower extremity revascularization using directional atherectomy: 12-month prospective results of the DEFINITIVE LE study. JACC Cardiovasc Interv 2014; 7: 923-33.

6. Sakakura K, Taniguchi Y, Yamamoto K, Wada H, Momomura SI, Fujita $\mathrm{H}$. When a burr can not penetrate the calcified lesion, increasing burr size as well as decreasing burr size can be a solution in rotational atherectomy. Int Heart J 2017; 58: 279-82.

7. Loehr SP, Hamilton C, Dyer R. Retrieval of entrapped guide wire in an IVC filter facilitated with use of a myocardial biopsy forceps and snare device. J Vasc Interv Radiol 2001; 12: 11169. 